\title{
Improved quality of life and reduced depressive symptoms in medical students after a single-session intervention
}

\author{
Mariane B. Bermudez, ${ }^{1}$ iD Monise Costanzi, ${ }^{1}$ Malu Joyce A. Macedo, ${ }^{1}$ iD Tiago Tatton-Ramos, ${ }^{2}$ \\ Alice C.M. Xavier, ${ }^{1}$ iD Ygor A. Ferrão, ${ }^{1}$ Kate H. Bentley, ${ }^{3}$ Gisele G. Manfro, ${ }^{2}$ Carolina B. Dreher ${ }^{1,2}$ \\ ${ }^{1}$ Universidade Federal de Ciências da Saúde de Porto Alegre, RS, Brazil. ${ }^{2}$ Universidade Federal do Rio Grande do Sul, Porto Alegre, RS, \\ Brazil. ${ }^{3}$ Harvard Medical School, Massachusetts General Hospital, Boston, MA, USA.
}

\begin{abstract}
Objective: Anxiety and depression are prevalent among medical students. Brazilian medical students have higher levels of depression and lower quality of life than their U.S. counterparts, and no preventive intervention exists for this risk group in Brazil. The Unified Protocol for Transdiagnostic Treatment of Emotional Disorders (UP), a cognitive-behavioral treatment protocol for neuroticism, was recently adapted into a single-session, preventive intervention. This study tested the impact of this protocol on psychiatric symptoms and quality of life in Brazilian medical students.

Methods: In this open trial, the intervention protocol was translated and adapted to Brazilian Portuguese. Medical students over 18 years of age without psychotic symptoms, severe depressive episodes, or acute psychiatric risk were included, undergoing a psychiatric clinical interview (MiniInternational Neuropsychiatric Interview [MINI]) and evaluation at baseline and at 7 and 30 days after a single-session UP that included experimental avoidance, quality of life, self-esteem, empathy, and anxiety symptom scales. A new evaluation was performed 90 days after the intervention.

Results: Sixty-two students participated. Ninety days after the intervention, there were significant reductions in the number of students who met the criteria for social anxiety disorder $(p=0.013)$ or panic disorder $(p=0.001)$. There were also significant improvements in depressive symptoms (Beck Depression Inventory, $\mathrm{p}<0.001$ ) and quality of life (Quality of Life Enjoyment and Satisfaction Questionnaire, $\mathrm{p}<0.001)$.

Conclusion: UP improved anxiety and depressive symptoms in medical students. The single-session group format could reduce costs and facilitate application. Future placebo-controlled studies are necessary to confirm these findings.
\end{abstract}

Keywords: Prevention; cognitive-behavioral therapy; unified protocol; medical students; quality of life

\section{Introduction}

Medical school is a long and arduous journey. It has been shown that psychological distress is greater in medical students than their age-matched peers. ${ }^{1,2}$ The high prevalence of anxiety and depression disorders in this group has been clearly established, ${ }^{3,4}$ and a study at the University of California found that $24 \%$ of medical students had depressive symptoms. ${ }^{5}$ The psychological distress experienced by medical students could be due to several potentially harmful effects of medical education, such as low levels of empathy, diminished academic performance, ${ }^{6}$ low professionalism, ${ }^{7,8}$ suboptimal patient care practices, ${ }^{2,9}$ and substance abuse. ${ }^{10}$ Several factors have been identified as possible causes of these findings, for example, the need to cope with patient suffering and death, lack of time, competitiveness, stringent academic demands and frequent examinations, all of which can decrease quality of life (QoL)

Correspondence: Mariane B. Bermudez, Rua Sarmento Leite, 245, CEP 90050-170, Porto Alegre, RS, Brazil.

E-mail: bagatinmariane@gmail.com

Submitted Apr 24 2019, accepted Aug 08 2019, Epub Dec 202019. among medical students. ${ }^{11-15}$ Additionally, psychological distress may play a part in psychopathology, which could contribute to suicidal behavior. Suicide rates among young people have increased over the past three decades. ${ }^{16}$ Downs et al. ${ }^{17}$ found that almost $8 \%$ of medical students met the criteria for high/significant suicide risk and that 10 of every 13 at-risk students were not receiving mental health treatment, indicating that there is a high proportion of untreated, at-risk, and potentially suicidal individuals in this population.

One Brazilian study reported that $24.9 \%$ of medical students experienced depressive symptoms while enrolled in medical school. ${ }^{18}$ Brazilian medical students have higher levels of depression, stress, and exhaustion, as well as less engagement in wellness behaviors and lower QoL than those in the USA. ${ }^{19}$ Damage to mental health is not only an issue for students: it can persist as a substantial component of a physician's life. Physicians
How to cite this article: Bermudez MB, Costanzi M, Macedo MJA, Tatton-Ramos T, Xavier ACM, Ferrão YA, et al. Improved quality of life and reduced depressive symptoms in medical students after a single-session intervention. Braz J Psychiatry. 2020;42:145-152. http://dx.doi.org/10.1590/1516-4446-2019-0526 
are a known risk group for suicide. In a recent metaanalysis, the suicide rate for female and male medical doctors was more than $200 \%$ and $40 \%$ higher, respectively, than that of women and men in other professions. ${ }^{20}$

Preventive mental health measures can prevent suffering, reduce inequality, create healthy and productive adults, and offer social and economic returns. ${ }^{21}$ Although there are different approaches, cognitive-behavioral therapy (CBT) is the most studied and effective approach to preventing mental health problems. ${ }^{22-24}$ A meta-analysis found that preventing psychiatric illness was not linked to protocol duration, since shorter interventions had the same effect size as longer ones. ${ }^{25}$ These findings suggest that brief interventions may also be effective, with the additional advantages of easier application and lower cost.

The Unified Protocol for Transdiagnostic Treatment of Emotional Disorders (UP) ${ }^{26}$ is a cognitive-behavioral intervention designed to address neuroticism, which has been considered a predictor of depressive and anxiety disorders. $^{27-30}$ The UP consists of eight treatment modules; the five "core" UP modules are designed to replace maladaptive and avoidant reactions through adaptive coping strategies. The UP has shown efficacy in treating anxiety, ${ }^{31,32}$ unipolar and bipolar depression, ${ }^{31,33}$ post-traumatic stress disorder, ${ }^{34}$ borderline personality disorder, ${ }^{35}$ nonsuicidal self-injury, ${ }^{36}$ alcohol abuse, ${ }^{37}$ and eating disorders. ${ }^{38}$ Recently, the developers of the UP adapted the original treatment protocol into a single-session, transdiagnostic intervention aimed at preventing emotional disorders in at-risk college students. ${ }^{39}$ The rationale for this brief, transdiagnostic preventive intervention was to deliver relevant strategies that are applicable across the full range of emotional experiences. Its single-session format can also facilitate cost-effectiveness and timeefficiency. In an initial pilot study, the modified preventive intervention had a small effect on neuroticism, QoL, and experiential avoidance in young adults at risk for emotional disorders. ${ }^{38}$ However, this data has not yet been replicated, and no studies with this CBT protocol have been performed in lower-income countries, such as Brazil. Therefore, this study aimed to adapt the intervention tested by Bentley et al. ${ }^{39}$ and examine its efficacy for promoting QoL and preventing anxiety and depressive symptoms in medical students, a high-risk group in Brazil.

\section{Methods}

\section{Study design and participants}

This was an open trial study. The students were recruited from two federal universities in southern Brazil through posters, Facebook, and institutional e-mails in the second semester of 2017. All medical students in both universities were invited to participate in the study. To take part, the students had to be: 1) over 18 years of age, and 2) willing to participate in the intervention. Students with psychotic symptoms, severe depressive episode, and acute psychiatric risk were excluded.

All participants were assessed individually with a structured psychiatric interview, followed by the Mini-International Neuropsychiatric Interview (MINI). ${ }^{40}$
The MINI is a short diagnostic interview for DSM-IV and ICD-10 psychiatric disorders that is applied by trained psychiatrists or psychiatry residents. Students who were diagnosed with any psychiatric disorder at the time of the interview were informed about their diagnosis and treatment options. Students who presented with psychotic symptoms, a manic episode, or a severe depressive episode were referred for treatment, and if they presented an acute psychiatric risk, they were referred to the psychiatric emergency unit. Students with suicidal ideation were excluded from the study because this brief intervention focused on mild-to-moderate symptoms. It is important to point out that aspects of suicidality were evaluated by both a psychiatrist and the MINI instrument.

The single-session preventive intervention was conducted in a classroom at each university in the second semester of 2017. Altogether, 62 students participated in the intervention, divided into eight groups of a maximum of 10 people. After the intervention, two students were excluded because they were unavailable for reassessment in the follow-up. Table 1 depicts the epidemiological and sociodemographic characteristics of the sample.

Table 1 Epidemiological and sociodemographic characteristics of the sample $(n=62)$

\begin{tabular}{|c|c|}
\hline Characteristics & \\
\hline \multicolumn{2}{|l|}{ Gender } \\
\hline Male & $25(40.3)$ \\
\hline Female & $37(59.7)$ \\
\hline Mean age & 23.43 \\
\hline \multicolumn{2}{|l|}{ Universities } \\
\hline 1 & $47(75.8)$ \\
\hline 2 & $15(24.2)$ \\
\hline \multicolumn{2}{|l|}{ Ethnicity } \\
\hline Caucasian & $49(79.0)$ \\
\hline Asian & $3(04.8)$ \\
\hline African & $10(16.1)$ \\
\hline \multicolumn{2}{|l|}{ Sexual orientation } \\
\hline Heterosexual & $51(82.3)$ \\
\hline Homosexual & $5(08.1)$ \\
\hline Bisexual & $6(09.7)$ \\
\hline \multicolumn{2}{|l|}{ Marital status } \\
\hline Single & $34(54.8)$ \\
\hline Married & $1(01.6)$ \\
\hline Dating & $27(43.5)$ \\
\hline \multicolumn{2}{|l|}{ Resides } \\
\hline Alone & $13(21.0)$ \\
\hline With family & $23(37.1)$ \\
\hline With friends & $26(41.9)$ \\
\hline Religion/spirituality & $36(58.1)$ \\
\hline \multicolumn{2}{|l|}{ Stage of the medical course } \\
\hline First or second year & $16(25.8)$ \\
\hline Third or fourth year & $31(50.0)$ \\
\hline Internship & $15(24.1)$ \\
\hline Family history of psychiatric illness & $29(46.8)$ \\
\hline
\end{tabular}

Data presented as $\mathrm{n}(\%)$, unless otherwise specified. 


\section{Procedure and measures}

The intervention was applied in person ${ }^{39}$ and focused on: 1) the functional nature of emotions; 2) mindful emotional awareness strategies; 3) cognitive flexibility; and 4) emotional avoidance and alternatives. ${ }^{39}$ It was designed as a single 2-hour workshop to be accessible to the greatest number of students in university centers. After the single-session intervention, one printable support worksheet was sent to the participants by e-mail each week for one month to remind them of the "take-away messages" and practical exercises in each module. The intervention was applied by two researchers: one was a psychiatrist trained in CBT and the other was a psychiatrist specializing in mindfulness.

The protocol was translated into Brazilian Portuguese by two independent researchers, and any discrepancies were further discussed and resolved by the other researchers. Final decisions were made after considering three key aspects: adhering to the original protocol, ensuring participant understanding, and prioritizing cultural relevance. The adaptation process also included evaluation of the UP video material, since each key concept in the original intervention ${ }^{39}$ is illustrated with a brief video. The suitability of the videos for each concept was discussed among the researchers. Research assistants, including medical students and psychiatry professors, evaluated the adequacy of the video for our target population. This first version was tested with a sample of 10 volunteer medical students who consented to participate.

After inclusion in the study, the baseline assessment was administered through self-applied questionnaires:

1) The Multidimensional Experiential Avoidance Questionnaire $(\mathrm{MEAQ})^{41}$ was used to evaluate the tendency to avoid uncomfortable thoughts or feelings It includes 62 items, with higher scores indicating more severe symptoms. This rating scale was translated into Portuguese. $^{42}$

2) The Quality of Life Enjoyment and Satisfaction Questionnaire Short Form (Q-LES-Q-SF), ${ }^{43}$ was used to assess the degree of satisfaction and pleasure in various areas of daily functioning. The Q-LES-Q-SF includes 16 items and has been translated and validated (Cronbach's alpha $=0.78$ ) into Portuguese. ${ }^{44}$

3) The Rosenberg Self-Esteem Scale (RSE) ${ }^{45}$ was used to evaluate self-esteem. The RSE consists of 10 items and has been validated for Brazilian Portuguese, ${ }^{46}$ showing good reproducibility.

4) The Interpersonal Reactivity Index (IRI) ${ }^{47}$ was used to measure affective, cognitive, and behavioral empathy. This scale consists of 21 items and has been validated (Cronbach's alpha $=0.75$ ) for Brazilian Portuguese. ${ }^{48}$

5) The Overall Anxiety Severity and Impairment Scale $(\mathrm{OASIS})^{49}$ was used to assess anxiety, including frequency, intensity, and behavioral and functional aspects. This instrument consists of five items and has also been validated for Brazilian Portuguese..$^{50}$

6) The Beck Depression Inventory (BDI) ${ }^{51}$ was used to evaluate depressive symptoms. It is a 21 -question inventory and has been validated (Cronbach's alpha = 0.81) for the Brazilian population. ${ }^{52}$ This baseline assessment was conducted up to 7 days before the intervention.

After the intervention session, the previously mentioned support material and exercises were e-mailed to the students each week for 1 month. Students diagnosed with a disorder could either begin psychotherapy or medication; they were questioned about these interventions in the follow-up interviews and these factors were controlled for in the statistical analysis. At 7 and 30 days after the intervention, the students were evaluated online using the MEAQ, Q-LES-Q, RSE, IRI, and OASIS scales. Ninety days after the intervention, in addition to these scales, a new psychiatric evaluation was performed with the BDI and MINI instruments. The students were also asked about improvement in subjective factors during this period with an unstructured questionnaire (physical activity, sleep quality, better use of leisure time, appetite/eating habits, family relationships, mindfulness, greater acceptance of emotions, study habits, participation in parties and events, academic performance, social life, procrastination, better cognitive flexibility, and drug use).

\section{Statistical analysis}

The statistical analysis was performed in SPSS version 20.0. Normality was tested with the Kolmogorov-Smirnov test. Little's MCAR test was used to control for missing data, which tests the null hypothesis that the missing data is missing completely at random (MCAR). If the $p$-value is less than 0.05 , the usual interpretation is that the missing data is not MCAR. Our results for Little's MCAR were $X^{2}=10.381$, Sig. $=0.663$. Student's $t$-test was used for normally distributed variables, and the Wilcoxon $U$ test was used for non-normally distributed variables. We used Pearson's and Fisher's chi-square tests and Yates tests to compare categorical variables. Analysis of variance, Mauchly's test, the Greenhouse-Geisser test, and posthoc Bonferroni were used to compare linear variables with normal distribution. Spearman's correlation coefficient was used for linear correlations between variables with non-normal distribution. Logistic regression (forward conditional method) was used to calculate associations between the variables. The significance level was set at $p<0.05$.

\section{Ethics statement}

All participants provided written informed consent prior to participation. This study is consistent with the Guidelines and Norms for Research Involving Human Beings (Resolution 466/12) and follows the ethical principles of the Declaration of Helsinki. The Universidade Federal de Ciências da Saúde de Porto Alegre and the Hospital de Clínicas de Porto Alegre ethics committee approved the study (no. 2.324.309). This study was registered in the Brazilian research database (CAAE: 7365.8817.3.1001. $5327)$ and in the Clinical Trials database (NCT03518411). 


\section{Results}

As shown in the flowchart (Figure 1), 76 students contacted the researchers about participation and 64 agreed to attend the initial evaluation (psychiatric interview plus scales). Two students were excluded: one due to acute psychiatric risk and one was unavailable for the session.

The frequency of psychiatric diagnoses before and after the intervention is presented in Table 2. Compared with baseline values, we observed clinical improvements in social anxiety disorder $(p=0.013)$ and panic disorder $(p=0.001)$ at 90 days after the UP. Fisher's test was performed to measure the potential impact of psychotherapy or use of psychiatric medication on this improvement and the results were not statistically significant.

We observed statistically significant improvement in depressive symptoms (BDI 11.64 vs. 7.82, $p<0.001$ ) and in QoL (Q-LES-Q 0.602 vs. 0.675, $\mathrm{p}<0.001$ ) in the self-report scales. These outcomes are described in Table 3 and illustrated in Figure 2. To analyze factors that may have influenced these findings, we stratified our sample into those who did or did not improve at least $10 \%$ in final BDI and Q-LES-Q scores compared to baseline. We carried out a bivariate comparison of gender, university, ethnicity, sexual orientation, marital status, living situation, religion/spirituality, semester, family history of psychiatric illness, psychotherapy, psychopharmacology, adherence to practical activities, the subjective improvement questionnaire, MINI psychiatric diagnoses, and BDI, Q-LES-Q, IRI, RSE and OASIS scores. Variables with a significant association $(p<0.10)$ were included in the logistic regression model (Table 4). Sexual orientation (heterosexual and homosexual), baseline BDI and OASIS scores, and diagnosis of bulimia were included as dependent variables for the QoL outcome (improvement in Q-LES-Q scores). The dependent variables included in the logistic regression model for reduced depressive symptoms (reduction in BDI scores) were living alone, living with relatives, and Q-LES-Q score. The students with the greatest reduction $\mathrm{BDI}$ scores lived alone or with family members. Regarding QoL, Q-LES-Q score improvement
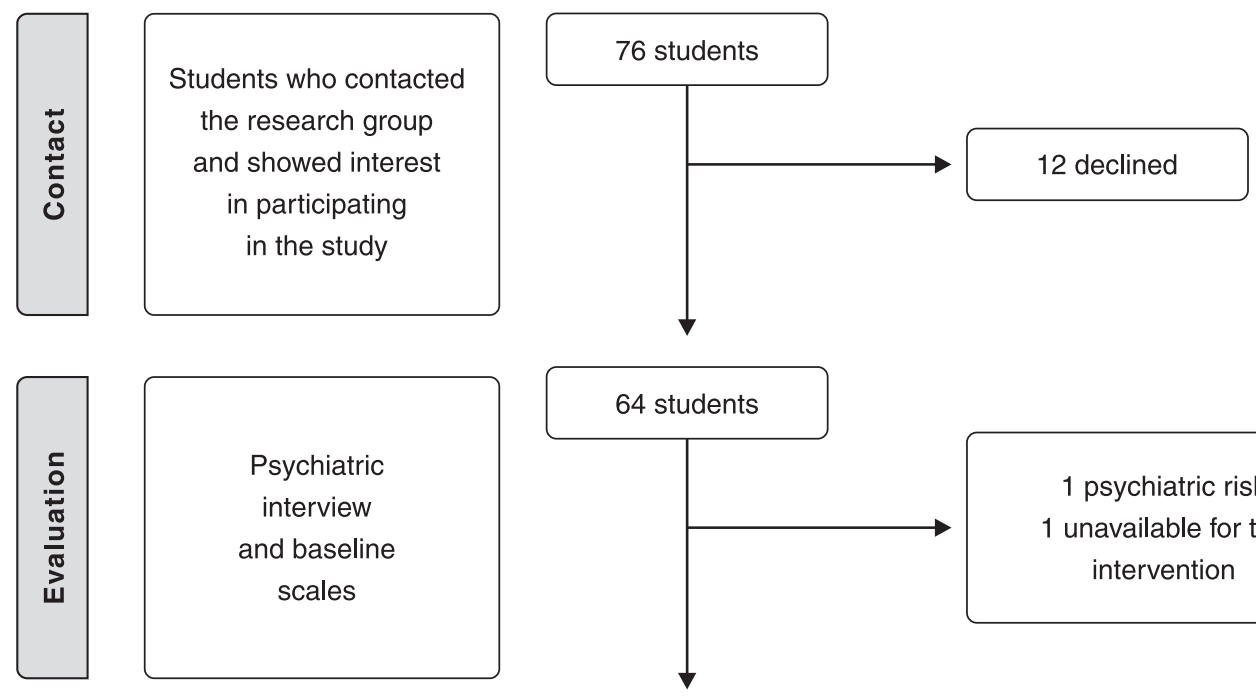

1 psychiatric risk

1 unavailable for the intervention
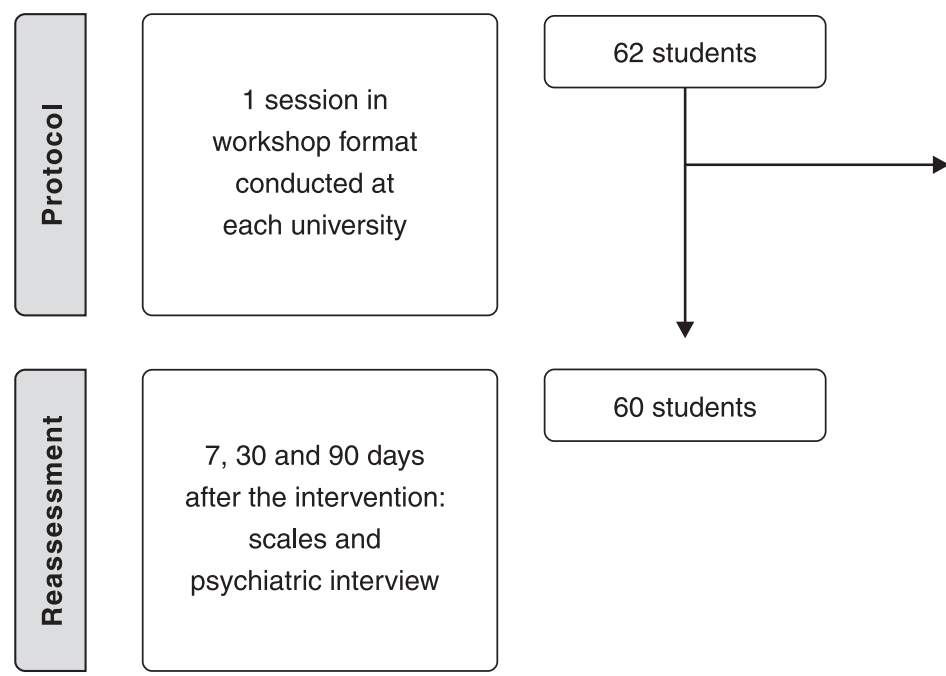

60 students

2 unavailable

for reassessment

Figure 1 Flowchart of students included in the study. 
Table 2 Psychiatric diagnoses according to the Mini-International Neuropsychiatric Interview $(n=60)$

\begin{tabular}{|c|c|c|c|c|c|}
\hline Diagnoses & Before UP & 90 days after UP & McNemar $p$-value & PT & $\mathrm{PP}$ \\
\hline Major depressive episode & $12(20.9)$ & $6(10.0)$ & 0.146 & 1.000 & 0.500 \\
\hline Dysthymia & $3(5.0)$ & $0(0)$ & 0.250 & 1.000 & 1.000 \\
\hline \multicolumn{6}{|l|}{ Drug dependence or abuse } \\
\hline Alcohol & $4(6.4)$ & $3(4.9)$ & 0.100 & 0.250 & 1.000 \\
\hline Cannabis & $5(8.0)$ & $2(3.3)$ & 0.375 & 1.000 & 1.000 \\
\hline LSD & $1(1.6)$ & $2(3.3)$ & 1.000 & 1.000 & 1.000 \\
\hline Ecstasy & $3(5.0)$ & $0(0)$ & 0.250 & 1.000 & 1.000 \\
\hline Hallucinogens & $2(3.3)$ & $2(3.3)$ & 1.000 & 1.000 & 1.000 \\
\hline Panic disorder & $11(18.0)$ & $0(0)$ & $0.001 *$ & 0.250 & 0.500 \\
\hline Agoraphobia & $9(15.0)$ & $4(6.7)$ & 0.227 & 1.000 & 1.000 \\
\hline Social anxiety disorder & $17(28.3)$ & $6(10.0)$ & $0.013^{*}$ & 0.500 & 1.000 \\
\hline Obsessive compulsive disorder & $5(8.0)$ & $6(9.9)$ & 1.000 & 1.000 & 1.000 \\
\hline Post-traumatic stress disorder & $1(1.6)$ & $1(1.6)$ & 1.000 & 1.000 & 1.000 \\
\hline Generalized anxiety disorder & $28(45.0)$ & $20(33.2)$ & 0.092 & 0.125 & 1.000 \\
\hline Bulimia & $4(6.7)$ & $0(0)$ & 0.125 & 1.000 & 1.000 \\
\hline
\end{tabular}

Data presented as $\mathrm{n}(\%)$.

PP = psychopharmacotherapy; PT = psychotherapy; UP = The Unified Protocol for Transdiagnostic Treatment of Emotional Disorders.

* Statistically significant.

Table 3 Dimensional variables at baseline and after the intervention

\begin{tabular}{lcccccc}
\hline & Before UP & 7 days after UP & 30 days after UP & 90 days after UP & $F$ & $p$-value \\
\hline MEAQ* & $187.80(34.44)$ & $173.17(32.60)$ & $165.95(39.45)$ & $196.10(25.52)$ & 50.14 & 0.028 \\
Q-LES-Q* & $0.602(0.150)$ & $0.630(0.163)$ & $0.659(0.160)$ & $0.675(0.154)$ & 8.61 & $<0.001^{*}$ \\
RSE* & $29.53(5.54)$ & $28.72(5.46)$ & $30.33(7.29)$ & $30.09(5.00)$ & 1.83 & 0.170 \\
OASIS* & $6.82(3.76)$ & $5.33(3.36)$ & $5.45(3.95)$ & $5.48(3.86)$ & 5.02 & 0.123 \\
IRI $^{\text {B }}$ & $72.08(7.57)$ & $72.20(7.60)$ & $72.19(7.19)$ & $71.92(7.38)$ & 0.026 & 0.97 \\
BDI $^{\dagger}$ median (min-max) & $10.5(0-31)$ & - & - & $7.0(0-26)$ & - & $<0.001^{*}$ \\
\hline
\end{tabular}

Data presented as median (SD), unless otherwise specified.

$\mathrm{BDI}=$ Beck Depression Inventory; IRI = Interpersonal Reactivity Index; MEAQ = Multidimensional Avoidance Questionnaire; Min-max = minimum and maximum values; OASIS = Overall Anxiety Severity and Impairment Scale; Q-LES-Q = Quality of Life Enjoyment and Satisfaction Questionnaire; RSE = Rosenberg Self-Esteem Scale; SD = standard deviation; UP = The Unified Protocol for Transdiagnostic Treatment of Emotional Disorders.

* Analysis of variance (ANOVA), Mauchly's test, and post-hoc Bonferroni.

Wilcoxon U test.

Statistically significant.

A Q-LES-Q scores

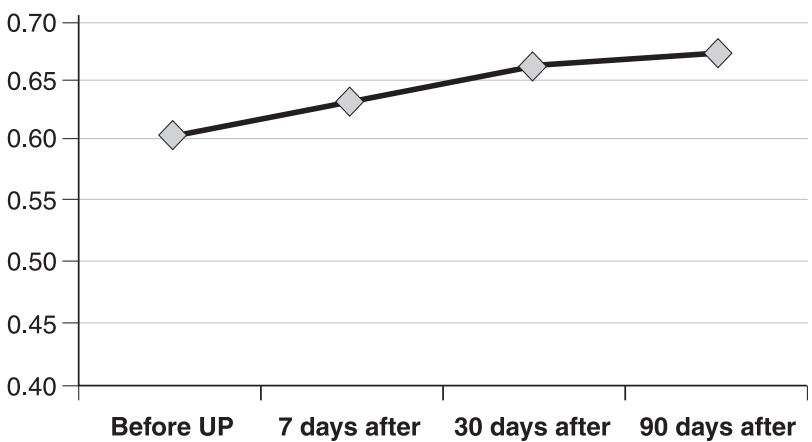

B BDI scores

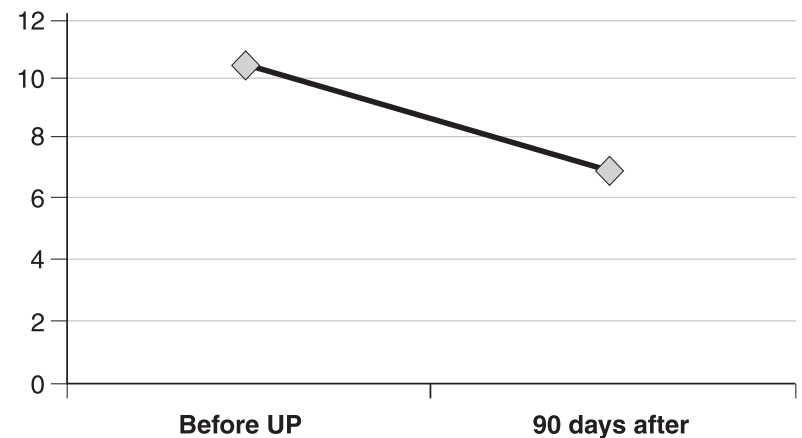

Figure 2 A) Quality of life improvement according to Quality of Life Enjoyment and Satisfaction Questionnaire (Q-LES-Q) score. B) Reduction of depressive symptoms according to Beck Depression Inventory (BDI) score. UP = The Unified Protocol for Transdiagnostic Treatment of Emotional Disorders.

was moderately influenced by reduced anxiety severity (OASIS, $p=0.034$ ) and depression symptoms (BDI, $p=0.005)$. Regarding the BDI outcome, students who lived alone $(p=0.007)$ or with relatives $(p=0.015)$ showed greater reduction of depressive symptoms than those who lived with friends. Furthermore, BDI score reduction was significantly related to $Q-L E S-Q$ score improvement $(p=0.001)$. 
Table 4 Logistic regression model for the Q-LES-Q and BDI outcomes

\begin{tabular}{lcccc}
\hline & & & \multicolumn{2}{c}{$95 \% \mathrm{Cl}$ to OR } \\
\cline { 4 - 5 } Q-LES-Q & $\mathrm{p}$-value & OR & Min & Max \\
\hline BDI & 0.005 & 0.069 & 0.011 & 0.438 \\
OASIS & 0.034 & 0.187 & 0.040 & 0.879 \\
Bulimia & 0.999 & 0.000 & 0.000 & \\
BDI & & & & \\
$\quad$ Lives alone & 0.032 & 7.566 & 1.190 & 48.17 \\
$\quad$ Lives with relatives & 0.040 & 4.866 & 1.071 & 22.102 \\
$\quad$ Q-LES-Q & $<0.001$ & 0.076 & 0.019 & 0.310
\end{tabular}

$95 \% \mathrm{Cl}=95 \%$ confidence interval; $\mathrm{BDI}=$ Beck Depression Inventory; Max = maximum values; Min = minimum values; OASIS = Overall Anxiety Severity and Impairment Scale; OR = odds ratio; Q-LES-Q = Quality of Life Enjoyment and Satisfaction Questionnaire.

Table 5 Subjective changes 90 days after the intervention $(\mathrm{n}=60)$

\begin{tabular}{ll}
\hline Changes & \\
\hline Greater acceptance of emotions & $44(73.3)$ \\
Better cognitive flexibility & $35(58.3)$ \\
Better mindfulness practice & $26(43.3)$ \\
Better family relationships & $18(30.0)$ \\
Better use of leisure time & $16(26.6)$ \\
Improved social life & $16(26.6)$ \\
Less procrastination & $16(26.6)$ \\
More physical activity & $15(25.0)$ \\
Better sleep quality & $12(20.0)$ \\
Improved study routine & $11(18.3)$ \\
Better academic performance & $11(18.3)$ \\
Improved appetite/eating & $7(11.6)$ \\
Participation in parties and events & $6(10.0)$ \\
Less drug use & $6(10.0)$ \\
\hline
\end{tabular}

Data presented as $\mathrm{n}(\%)$.

It should be pointed out that the onset of pharmacological or psychotherapeutic treatment, as well as compliance with the e-mailed activities, had no significant influence on these outcomes.

In the subjective assessment of whether the intervention helped improve some aspect of life (on a scale of 1 to $5: 1=$ not at all, and $5=$ completely), $79.8 \%$ of the students responded with three or more points. The student-defined subjective changes are described in Table 5.

\section{Discussion}

This study translated and adapted a preventive intervention based on the UP ${ }^{32,39}$ for use with Brazilian medical students. The students rated the intervention as highly acceptable and most reported that it was beneficial. There was statistically significant improvement in BDI and Q-LES-Q scores and in the prevalence of panic disorder and social anxiety disorder. It should be pointed out that most of the participants had symptoms of depression and anxiety (although we did not select for elevated levels of anxiety or depression) and, surprisingly, approximately $78 \%$ of the sample had some psychiatric disorder. This high prevalence of psychiatric symptoms and disorders could be explained by the fact that the Brazilian population is exposed to stressful socioeconomic-related situations. ${ }^{53}$ Another point to consider is that access is difficult to specialized public health services such as psychiatry or psychotherapy, ${ }^{54}$ so treatment-seeking students may have been especially interested in the study.

We found that most of the medical students who participated in this study learned new concepts and new skills. Curiously, the curriculum of medical disciplines encompasses medical psychology and psychiatry, so our sample consists of participants with a certain level of psychoeducation on mental health conditions. Thus, although behavioral sciences have been taught for a considerable time in the medical curriculum, it seems that they do not meet the personal needs of students. More than $70 \%$ of our subjects responded that they could deal more appropriately with their emotions after the intervention.

Our intervention was focused on preventing mental disorders by reducing subsyndromal symptoms. It was effective in reducing depressive symptoms according to $\mathrm{BDI}$ scores. The reduction in BDI scores was greater among students who lived alone or with family members. Perhaps those who lived with colleagues had already experienced, through the socialization process, the benefits of a group intervention. A previous prevention program that was effective in reducing depressive symptoms in adolescents specifically emphasized the importance of the social network, and the participants remained at a low level of depressive symptoms due to strong social networks. ${ }^{55}$ In the present study, it could be that participating in a group with colleagues and sharing aspects of their mental health had a positive impact, particularly for those who did not have a strong social network. However, we could not analyze the group effect in our study due to the open trial design.

According to the Q-LES-Q scores, the participants' QoL improved after the intervention, and the variation in $Q$ LES-Q improvement was associated with the change in $\mathrm{BDI}$ and OASIS scores. This finding is in accordance with the literature, ${ }^{56-60}$ which reports that there is a strong inverse correlation between QoL and depressive symptoms. However, we did not find a statistically significant reduction in anxiety symptoms after the intervention. This might be due to the fact that baseline OASIS scores were below the cutoff point for anxiety prior to application of the protocol, which could have made it difficult to observe changes in this outcome.

There was a reduction in the frequency of psychiatric diagnoses after the intervention, mainly regarding panic disorder and social anxiety disorder. These outcomes might be due to the fact that most participants had mild symptoms (OASIS $<8$, mild anxiety). Presumably, a brief education-style workshop in the UP format was effective for both preventing and treating these disorders. It could be that that the participants recovered because the natural history of these disorders has a wax-and-wane pattern. In addition, the timing of the intervention and follow-up assessments during the academic year could 
have impacted our findings. To minimize this limitation, the single-session intervention was delivered weekly over a period of 2 months with students from different classes.

There was no significant change in RSE scores. This might have occurred because self-esteem is a long-term aspect and interferes with personality characteristics. ${ }^{61}$ Additionally, there was no improvement in empathy (IRI). Although the protocol was not focused on empathic issues, this variable was incorporated to assess whether reducing psychiatric symptoms would promote empathy. Meanwhile, MEAQ scores had improved significantly at 7 and 30 days after the intervention. Nevertheless, the improvement disappeared by the 90-day follow-up. Perhaps more frequent or intensive interventions might be needed for more significant findings.

The fact that $80 \%$ of the students reported that they had benefited from the intervention reinforces the importance of making preventive interventions for psychiatric disorders systematically available in the academic environment for medical students. However, our results should be interpreted in light of some limitations. Ours was a convenience sample, which could have created a selection bias, i.e., students without psychiatric symptoms might not have felt motivated to participate in the intervention. Similarly, students with a mental illness associated with social impairment might have avoided participation. Moreover, it was an open study with no control group, and no comparisons were made between subjects undergoing individual therapy and those on a waiting list. Furthermore, due to the scarcity of psychoeducation about emotions and feelings in Brazil, simply meeting and conversing about the subject might have benefitted the students.

Although the majority of the disease burden associated with mental illness is attributed to disability rather than mortality, these disorders have a large economic impact, including lost productivity, career/family costs, and government expenditure. ${ }^{62}$ Preventing mental disorders among young people is one of the soundest investments a society could make, and the benefits include higher productivity, lower treatment costs, less suffering and premature mortality, more cohesive families, and better adjusted and more successful people. ${ }^{63}$ This study concludes that the UP was an acceptable and feasible intervention for preventing mental disorders in a risk group. The single 2-hour session transdiagnostic group format of reduces financial costs and facilitates the program's dissemination. Further studies are required on measures for preventing mental disorders, including randomized controlled trials.

\section{Disclosure}

The authors report no conflicts of interest.

\section{References}

1 Dahlin M, Joneborg N, Runeson B. Stress and depression among medical students: a cross-sectional study. Med Educ. 2005;39: 594-604.

2 Dyrbye LN, Thomas MR, Shanafelt TD. Systematic review of depression, anxiety, and other indicators of psychological distress among U.S. and Canadian medical students. Acad Med. 2006;81: 354-73.

3 Rotenstein LS, Ramos MA, Torre M, Segal JB, Peluso MJ, Guille C, et al. Prevalence of depression, depressive symptoms, and suicidal ideation among medical students: a systematic review and metaanalysis. JAMA. 2016;316:2214-36.

4 Brenneisen Mayer F, Souza Santos I, Silveira PS, Itaqui Lopes MH, de Souza AR, Campos EP, et al. Factors associated to depression and anxiety in medical students: a multicenter study. BMC Med Educ. 2016;16:282.

5 Givens JL, Tjia J. Depressed medical students' use of mental health services and barriers to use. Acad Med. 2002;77:918-21.

6 Clark DC, Zeldow PB. Vicissitudes of depressed mood during four years of medical school. JAMA. 1988;260:2521-8.

7 Dyrbye LN, Massie FS Jr, Eacker A, Harper W, Power D, Durning SJ, et al. Relationship between burnout and professional conduct and attitudes among US medical students. JAMA. 2010;304:1173-80.

8 Dyrbye LN, West CP, Satele D, Boone S, Sloan J, Shanafelt TD. A national study of medical students' attitudes toward self-prescribing and responsibility to report impaired colleagues. Acad Med. 2015; 90:485-93.

9 Dyrbye LN, Thomas MR, Shanafelt TD. Medical student distress: causes, consequences, and proposed solutions. Mayo Clin Proc. 2005;80:1613-22.

10 Jackson ER, Shanafelt TD, Hasan O, Satele DV, Dyrbye LN. Burnout and alcohol abuse/dependence among U.S. medical students. Acad Med. 2016;91:1251-6.

11 Tempski P, Santos IS, Mayer FB, Enns SC, Perotta B, Paro HB, et al. Relationship among medical student resilience, educational environment and quality of life. PLoS One. 2015;10:e0131535.

12 MacLeod RD, Parkin C, Pullon S, Robertson G. Early clinical exposure to people who are dying: learning to care at the end of life. Med Educ. 2003;37:51-8.

13 Tempski P, Bellodi PL, Paro HB, Enns SC, Martins MA, Schraiber LB. What do medical students think about their quality of life? A qualitative study. BMC Med Educ. 2012;12:106.

14 Wear D. "Face-to-face with It": medical students' narratives about their end-of-life education. Acad Med. 2002;77:271-7.

15 Wolf TM. Stress, coping and health: enhancing well-being during medical school. Med Educ. 1994;28:8-17.

16 Beautrais AL. Risk factors for suicide and attempted suicide among young people. Aust New Zeal J Psychiatry. 2000;34:420-36.

17 Downs N, Feng W, Kirby B, McGuire T, Moutier C, Norcross W, et al. Listening to depression and suicide risk in medical students: the healer education assessment and referral (HEAR) program. Acad Psychiatry. 2014;38:547-53.

18 de Vasconcelos TC, Dias BRT, Andrade LR, Melo GF, Barbosa L, Souza E. Prevalência de sintomas de ansiedade e depressão em estudantes de medicina. Rev Bras Educ Med. 2015;39:135-42.

19 Lucchetti G, Damiano RF, DiLalla LF, Lucchetti AL, Moutinho IL, da Silva Ezequiel $\mathrm{O}$, et al. Cross-cultural differences in mental health, quality of life, empathy, and burnout between US and Brazilian medical students. Acad Psychiatry. 2018;42:62-7.

20 Schernhammer ES, Colditz GA. Suicide rates among physicians: a quantitative and gender assessment (meta-analysis). Am J Psychiatry. 2004;161:2295-302.

21 Langford R, Bonell CP, Jones HE, Pouliou T, Murphy SM, Waters E, et al. The WHO health promoting school framework for improving the health and well-being of students and their academic achievement. Cochrane database Syst Rev. 2014;(4):CD008958.

22 Dvořáková K, Kishida M, Li J, Elavsky S, Broderick PC, Agrusti MR, et al. Promoting healthy transition to college through mindfulness training with first-year college students: pilot randomized controlled trial. J Am Coll Heal. 2017;65:259-67.

23 Pistorello J, Fruzzetti AE, Maclane C, Gallop R, Iverson KM. Dialectical behavior therapy (DBT) applied to college students: a randomized clinical trial. J Consult Clin Psychol. 2012;80:982-94.

24 Vazquez FL, Torres A, Blanco V, Diaz O, Otero P, Hermida E. Comparison of relaxation training with a cognitive-behavioural intervention for indicated prevention of depression in university students: a randomized controlled trial. J Psychiatr Res. 2012;46: 1456-63.

25 Fisak BJ Jr, Richard D, Mann A. The prevention of child and adolescent anxiety: a meta-analytic review. Prev Sci. 2011;12:255-68. 
26 Wilamowska ZA, Thompson-Hollands J, Fairholme CP, Ellard KK, Farchione TJ, Barlow DH. Conceptual background, development, and preliminary data from the unified protocol for transdiagnostic treatment of emotional disorders. Depress Anxiety. 2010;27:882-90.

27 Genetics of Personality Consortium, de Moor MH, van den Berg SM, Verweij KJ, Krueger RF, Luciano M, et al. Meta-analysis of genomewide association studies for neuroticism, and the polygenic association with major depressive disorder. JAMA Psychiatry. 2015; 72:642-50.

28 Everaerd D, Klumpers F, van Wingen G, Tendolkar I, Fernandez G. Association between neuroticism and amygdala responsivity emerges under stressful conditions. Neuroimage. 2015;112:218-24.

29 Ormel J, Jeronimus BF, Kotov R, Riese H, Bos EH, Hankin B, et al. Neuroticism and common mental disorders: meaning and utility of a complex relationship. Clin Psychol Rev. 2013;33:686-97.

30 Spinhoven P, Penelo E, de Rooij M, Penninx BW, Ormel J. Reciprocal effects of stable and temporary components of neuroticism and affective disorders: results of a longitudinal cohort study. Psychol Med. 2014;44:337-48.

31 Farchione TJ, Fairholme CP, Ellard KK, Boisseau CL, ThompsonHollands J, Carl JR, et al. Unified protocol for transdiagnostic treatment of emotional disorders: a randomized controlled trial. Behav Ther. 2012;43:666-78.

32 Barlow DH, Farchione TJ, Bullis JR, Gallagher MW, Murray-Latin H, Sauer-Zavala $S$, et al. The unified protocol for transdiagnostic treatment of emotional disorders compared with diagnosis-specific protocols for anxiety disorders: a randomized clinical trial. JAMA Psychiatry. 2017;74:875-84.

33 Boswell JF, Anderson LM, Barlow DH. An idiographic analysis of change processes in the unified transdiagnostic treatment of depression. J Consult Clin Psychol. 2014;82:1060-71.

34 Gallagher MW. The unified protocol for posttraumatic stress disorder. In: Barlow DH, Farchione T. Applications of the unified protocol for transdiagnostic treatment of emotional disorders. Oxford: Oxford University; 2017. p. 111-26.

35 Sauer-Zavala S, Bentley KH, Wilner JG. Transdiagnostic treatment of borderline personality disorder and comorbid disorders: a clinical replication series. J Pers Disord. 2016;30:35-51.

36 Bentley KH, Nock MK, Sauer-Zavala S, Gorman BS, Barlow DH. A functional analysis of two transdiagnostic, emotion-focused interventions on nonsuicidal self-injury. J Consult Clin Psychol. 2017; 85:632-46.

37 Ciraulo DA, Barlow DH, Gulliver SB, Farchione T, Morissette SB, Kamholz BW, et al. The effects of venlafaxine and cognitive behavioral therapy alone and combined in the treatment of co-morbid alcohol use-anxiety disorders. Behav Res Ther. 2013; 51:729-35.

38 Thompson-Brenner H, Boswell JF, Espel-Huynh H, Brooks G, Lowe MR. Implementation of transdiagnostic treatment for emotional disorders in residential eating disorder programs: a preliminary pre-post evaluation. Psychother Res. 2019;29:1045-61.

39 Bentley KH, Boettcher H, Bullis JR, Carl JR, Conklin LR, SauerZavala $\mathrm{S}$, et al. Development of a single-session, transdiagnostic preventive intervention for young adults at risk for emotional disorders. Behav Modif. 2018;42:781-805.

40 Amorim P. Mini International Neuropsychiatric Interview (MINI): validação de entrevista breve para diagnóstico de transtornos mentais. Braz J Psychiatry. 2000;22:106-15.

41 Gamez W, Chmielewski M, Kotov R, Ruggero C, Watson D. Development of a measure of experiential avoidance: the multidimensional experiential avoidance questionnaire. Psychol Assess. 2011;23: $692-713$.

42 Costa FMA, Cappi C, Hoexter MQ. Experiências adversas na infância e adolescência, mediadores e transtorno obsessivo-compulsivo: um estudo com pacientes, irmãos e controles [dissertação]. São Paulo: Universidade de São Paulo; 2017. https://doi.org/10.11606/ D.5.2019.tde-16042019-100553
43 Endicott J, Nee J, Harrison W, Blumenthal R. Quality of life enjoyment and satisfaction questionnaire: a new measure. Psychopharmacol Bull. 1993;29:321-6.

44 Zubaran C, Foresti K, Thorell MR, Franceschini PR, Homero W Portuguese version of the quality of life enjoyment and satisfaction questionnaire: a validation study. Rev Panam Salud Publica. 2009; 25:443-8.

45 Rosenberg M. Conceiving the self. New York: Basic Books; 1979.

46 Dinil GM, Quaresma MR, Ferreira LM. Translation into portuguese, cultural adaptation and validation of the Rosenberg self-esteem scale. Rev Bras Cir Plast. 2004;19:42-52.

47 Davis $\mathrm{MH}$. Measuring individual differences in empathy: evidence for a multidimensional approach. J Pers Soc Psychol. 1983;44:113-26.

48 Koller SH, Camino C, Ribeiro J. Adaptação e validação interna de duas escalas de empatia para uso no Brasil. Estud Psicol. 2001;18:43-53.

49 Norman SB, Cissell SH, Means-Christensen AJ, Stein MB. Development and validation of an Overall Anxiety Severity And Impairment Scale (OASIS). Depress Anxiety. 2006;23:245-9.

50 Koller SH, DeSousa DA, Salum GA. The THEMIS statement: a Brazilian multimodal experience to the promotion of evidencebased practice in the assessment of anxiety disorders. Acta Psychopathol. 2016;219.

51 Beck AT, Ward CH, Mendelson M, Mock J, Erbauch J. An inventory for measuring depression. Arch Gen Psychiatry. 1961;4:561-71.

52 Gorenstein C, Andrade L. Validation of a Portuguese version of the Beck Depression Inventory and the state-trait anxiety inventory in Brazilian subjects. Braz J Med Biol Res. 1996;29:453-7.

53 Barros FC, Matijasevich A, Santos IS, Horta BL, da Silva BG, Munhoz TN, et al. Social inequalities in mental disorders and substance misuse in young adults: a birth cohort study in Southern Brazil. Soc Psychiatry Psychiatr Epidemiol. 2018;53:717-26.

54 Amaral CE, Onocko-Campos R, de Oliveira PR, Pereira MB, Ricc $E C$, Pequeno $M L$, et al. Systematic review of pathways to mental health care in Brazil: narrative synthesis of quantitative and qualitative studies. Int J Ment Health Syst. 2018;12:65.

55 Possel P, Baldus C, Horn AB, Groen G, Hautzinger M. Influence of general self-efficacy on the effects of a school-based universal primary prevention program of depressive symptoms in adolescents: a randomized and controlled follow-up study. J Child Psychol Psychiatry. 2005;46:982-94.

56 Agrawal N, Bird JS, von Oertzen TJ, Cock H, Mitchell AJ, Mula M. Depression correlates with quality of life in people with epilepsy independent of the measures used. Epilepsy Behav. 2016;62:246-50.

57 Kanner AM, Barry JJ, Gilliam F, Hermann B, Meador KJ. Anxiety disorders, subsyndromic depressive episodes, and major depressive episodes: do they differ on their impact on the quality of life of patients with epilepsy? Epilepsia. 2010;51:1152-8.

58 Lin $\mathrm{CH}$, Yen YC, Chen MC, Chen CC. Depression and pain impair daily functioning and quality of life in patients with major depressive disorder. J Affect Disord. 2014;166:173-8.

59 Raab PA, Mackintosh MA, Gros DF, Morland LA. Impact of comorbid depression on quality of life in male combat Veterans with posttraumatic stress disorder. J Rehabil Res Dev. 2015;52:563-76.

60 Sainsbury K, Mullan B, Sharpe L. Reduced quality of life in coeliac disease is more strongly associated with depression than gastrointestinal symptoms. J Psychosom Res. 2013;75:135-41.

61 Skorek M, Song AV, Dunham Y. Self-esteem as a mediator between personality traits and body esteem: path analyses across gender and race/ethnicity. PLoS One. 2014;9:e112086.

62 Mihalopoulos C, Vos T, Pirkis J, Carter R. The economic analysis of prevention in mental health programs. Annu Rev Clin Psychol. 2011;7:169-201.

63 Dernovsek MZ, Sprah L. A preliminary report on the implementation of the European Service and Mapping Schedule (ESMS) in Slovenia to map preventive and promotion activities in mental health. Psychiatr Danub. 2008;20:141-7. 